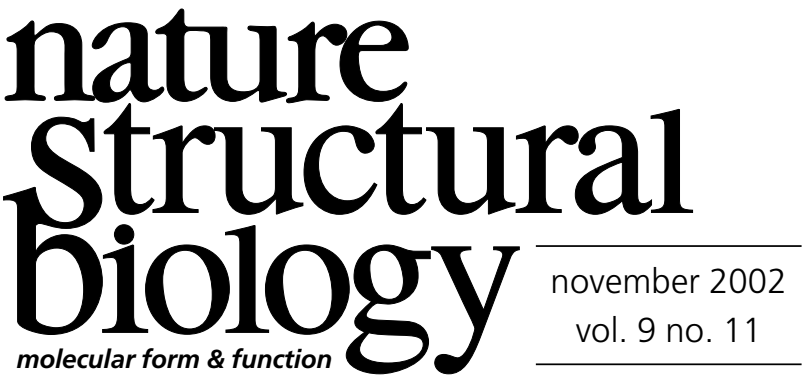

\title{
The times they are e-changing
}

Recently, Nature Structural Biology launched a new online manuscript submission system that most of us at the journal have come to refer to as 'EJP'. EJP stands for e-Journal Press, the software developers who worked with Nature Publishing Group to design the system. Through colloquial usage, however, 'EJP' has already become something of a buzzword for us, as in: "Please ask the author to load the text and figures into EJP", "Could you check in EJP to see how many manuscripts we've received this month?" or "I'm feeling a little under the weather, but I'd really like to find referees for this manuscript, maybe I'll just log on to EJP from home."

What makes EJP such an improvement over our previous manuscript handling system is that it allows us to do all these things and more. Using the new system, authors can submit their manuscripts electronically, directly uploading text and figures of several possible formats via a secure website. The files are combined and converted into a single PDF file that is available to editors and the chosen referees once they are signed into the password-protected site.

Electronic submission is rapidly becoming standard in the publishing industry, and there are obvious advantages to such a system that have driven its widespread implementation. Electronic submission eliminates the necessity for multiple paper copies of manuscripts, as well as the expense of postal and express mail services. In addition to cutting costs and reducing media wastage, electronic submission saves time. Once submitted, manuscripts are instantly available to the relevant editors and referees; authors are able to track the status of their manuscript directly over the internet. And, while referees (or editors!) may be reluctant to lug a stack of manuscripts home or while traveling, in EJP manuscripts can be accessed wherever there is an internet connection.

The specific advantages of EJP as an electronic submission system are perhaps a little less obvious until you start navigating your way through the system. To do this, simply go to the Nature Structural Biology website. We've added a new icon, so one click will take you directly to the sign-in page. Referees and authors that were present in our previous database have automatically been registered and assigned a username and temporary password in the new system. If you have not yet received a username and temporary password, these will be assigned once you complete the user registration form. When signing into the system for the first time, you will be asked to change your password (for security reasons); this would also be a good time to update and/or verify your user profile. Providing complete and accurate information - including providing keywords to highlight your area(s) of expertise and interest - is one of the ways you can help us to help you get the most out of EJP. Once inside EJP, the software guides authors through the submission process - and referees through the process of accessing manuscripts and submitting reviews - with ease.

Our own editorial procedures should become much more efficient thanks to EJP. Built into the software is an internal manuscript tracking system, which keeps tabs on all the manuscripts and generates task lists for each editor, providing a reminder of which manuscripts need attention and what needs to be done, and allowing us to prioritize our workloads accordingly. Additionally, some of the enhanced features of EJP, such as utilizing those keywords you provided to match suitable referees to manuscripts and using the statistics kept by the system to identify consistently good referees, are anticipated to speed up manuscript handling.

As with most technological improvements, you may occasionally (we hope rarely) encounter problems. If this happens, or if you have suggestions as to how we might improve the system to serve the community better, please feel free to contact us (email: nsb@natureny.com).

The thing about buzzwords is that they have a way of working their way into our common language, especially in these technologically fluid times. It wasn't so long ago that people considered words like internet and website to be the preserve of the tech savvy; now these words have made their way into the Oxford English Dictionary. While we don't think EJP is destined for the pages of the OED, we are enthusiastic about its future at NSB. 